\title{
German Plural Processing: New Evidence from ERP- and Reaction Time Experiments
}

\author{
Verena Winter ${ }^{1}$, Carsten Eulitz ${ }^{1}$, Tanja Rinker ${ }^{1,2}$ \\ ${ }^{1}$ Department of Linguistics, University of Konstanz, Konstanz, Germany \\ ${ }^{2}$ Zukunftskolleg, University of Konstanz, Konstanz, Germany \\ Email: tanja.rinker@uni-konstanz.de
}

Received 27 July 2013; revised 2 September 2013; accepted 10 September 2013

Copyright (C) 2014 by authors and Scientific Research Publishing Inc.

This work is licensed under the Creative Commons Attribution International License (CC BY). http://creativecommons.org/licenses/by/4.0/ (c) (i) Open Access

\begin{abstract}
The processing of the German plural has been examined in a range of behavioral and neurophysiological studies. A number of studies so far showed differences between the processing of the default plural form -s and the irregular plural form -(e)n. While previous studies focused on the examination of generally two plural categories (e.g. -(e)n versus -s), the current study aimed at examining four German plural categories with different morpheme endings and violating them cross-wise. The results of the current auditory event-related (ERP) and reaction time study show general similarities between the more acoustically salient plural categories. In the early latency range from $200-400 \mathrm{~ms}-\mathrm{n}$ violated by -s plural (and the cross-condition) act differently from the -e versus -er plural violations (and the cross-conditions) but in the later time windows only violation effects are visible. Processing differences may be explained by the morphological characteristics of the plural categories and/or phonetic differences between the suffixes.
\end{abstract}

\section{Keywords}

German Plural; Event-Related Potentials; Reaction Time Experiment

\section{Introduction}

The German plural is marked by five plural suffixes (-s, -(e)n, -er, -e and the zero-plural). Some masculine words of the categories -e, and the zero-allomorph and most of the words of the category -er mark the plural by an umlaut alternation in the stem. The allocation of the different plural morphemes to the stems is determined by morpho-phonological and prosodic constraints, and is linked to specific gender features (Wiese, 2009).

According to the Dual Mechanism Model (DMM), the processing of regularly and irregularly inflected forms is fundamentally different. Irregular inflectional forms are stored as a whole in the mental lexicon, whereas reg- 
ular inflectional forms are processed in a rule-based manner (Pinker \& Prince, 1992). The DMM was originally based on observations for the English past tense which can be divided into regular and irregular types. Regular suffixation with -ed is applied to the majority of verbs and it is used as a default for novel words and pseudo words. More recently, the DMM has also been applied to German plural formation. Here, the division is not as clear-cut as the so-called regular $-\mathrm{s}$ is not used for the majority of nouns, yet $-\mathrm{s}$ is considered the default that can be applied e.g. to novel nouns, proper names, nominalized conjunctions, etc. Nonetheless, while being the least frequent among the plural forms in German it is the most productive (Marcus et al., 1995). Previous psycholinguistic experiments have confirmed the special status of plural -s (for a review, see Clahsen, 1999). Since, according to the DMM, pluralization with -s is formed "on the spot", it was found not be affected by frequency effects unlike other plural forms (Penke \& Krause, 2002).

Additional support for the DMM comes from an electrophysiological study by Weyerts et al. (1997). Here, words with correct and incorrect plural morphemes were visually presented in a sentence with the critical (correct/incorrect) noun at the end. The -s plural (items were loanwords and proper names) and the -(e)n plural (divided into feminine nouns with schwa ending and masculine/neuter nouns) were compared. It was expected that violations with -s ("regularization") would elicit a left anterior negativity (LAN) (Kluender \& Kutas, 1993), reflecting the detection of a grammatical violation whereas violations with -(e)n ("irregularization") would elicit a central negativity (N400, Kutas \& Hillyard, 1980). The plural violation with -s was reflected by a LAN (irrespective of the gender of the items), whereas the violation with the irregular plural form -(e)n was reflected by an N400, as predicted on the bases of the DMM-model.

Lück et al. (2006) used a similar paradigm in the auditory domain. Contrary to Weyerts et al. (1997), the critical noun was presented embedded in a sentence. The result showed a small central negativity for the irregularization but additionally, a reanalysis-component (P600) was observed in the violated -s condition. Crucially, a LAN was also evoked by the irregularization with -(e)n. It was suggested that the differences between the studies are due to the auditory versus the visual presentation. In the auditory design the word plus violation is processed in a serial manner whereas in the visual domain it can be processed as a whole. The surname condition only elicited an N400 which can be considered to be a fundamentally different process as a lexical violation is evoked.

The aim of the present study was to extend the previous studies with respect to the German plural system by using all plural categories (except for the zero-plural for methodological reasons). In line with the study by Lück et al. (2006) it was expected that all plural violations would elicit a negativity over anterior brain regions, followed by a P600. If the predictions of the DMM were to hold for the processing of the German plural in the auditory domain, differences between "regular"/“irregular" plural types with respect to an additional central negativity would be expected. With respect to the reaction time experiment, a division between the regular and irregular plural types should be reflected in different reaction times between plural categories.

\section{Methods}

\subsection{Subjects}

24 (15 females) German native speakers participated in the reaction time experiment, their ages ranging between 21 and 27 with mean age of 23.4 years (SD = 2.4). For the ERP-experiment, 28 (17 females) German native speakers participated, their ages ranging between 21 and 28 with mean age of 23.6 years $(S D=2.7)$. All participants were right-handed according to the Edinburgh Handedness Inventory (Oldfield, 1971) and had normal hearing and eyesight, none of the subjects reported any neurological disorders. All were university students of various disciplines and received monetary compensation for their partication. The study was approved by the Ethics Board of the University of Konstanz and conducted according to the Declaration of Helsinki.

\subsection{Materials}

The majority of the items used in the study were very frequent nouns taken from a parental language questionnaire for two-year old children and included only depictable items (as the material was also used in a subsequent study with child language learners (Rinker et al., 2011)).

The German nouns were embedded in noun phrases following the pattern quantifier + noun + plural ending, e.g. viele Affe-n (“many monkeys"). Four correct conditions (-(e)n, -s, -e, -er) and four crosswise incorrect conditions (-(e)n viol. with -s; -s viol. with -(e)n; -er viol. with -e; -e viol. with -er) were created with 40 items each 
and 160 items in total.

\section{Study I: Reaction Time Experiment}

\subsection{Procedure}

Participants sat in a dimly lit and sound attenuating room. The items were presented acoustically through headphones. A white cross $(2 \times 2 \mathrm{~cm})$ on a black background was shown on a computer screen as a fixation aid. Subjects were instructed to decide if the phrase was correct or incorrect with regard to its grammaticality. They were told to respond immediately after the presentation of the item by button press. The thumbs of the left and right hand rested on two buttons of a button box. The function of each button was fixed across all subjects, with pushing the right button in answer to a correct and left to an incorrect plural ending. Items lasted $936 \mathrm{~ms}$ (SD = $100 \mathrm{~ms}$ ) on average. The interstimulus interval (ISI) varied between 3000 and $5000 \mathrm{~ms}$. The experiment lasted 45 min with breaks and a preceding practice phase with 20 items.

Responses were calculated from the deviation point, i.e. the point where a morpheme can be identified as correct or incorrect. The deviation points were individually set for the different items. Responses below $100 \mathrm{~ms}$ and above 1000 ms were cut off (Luce, 1986; Ratcliff, 1993) and incorrect responses were excluded. This led to the exclusion of $8.71 \%$ of the responses. Reaction time was subsequently analyzed.

\subsection{Reaction Time Results}

A main effect could be observed for plural type $(\mathrm{F}(1,23)=44.6, p<.001)$ and for violation $(\mathrm{F}(1,23)=6.2, p$ $=.016)$. The interaction of plural type and violation $(\mathrm{F}(2,46)=31.2, p<.001)$ was statistically significant as well.

Post hoc-analysis with pairwise t-tests between the correct plurals show that the response to the -(e)n plural was faster than to the -er plural $(\mathrm{t}(24)=-4.018 ; p<.001)$, but no difference was found between the response to the $-\mathrm{s}$ plural $(\mathrm{t}(24)=1.031 ; p=.313)$ and $-\mathrm{e}$ plural $(\mathrm{t}(24)=.038 ; p=.970)$. The $-\mathrm{e}$ plural was processed faster than the -er plural $(\mathrm{t}(24)=-3.141 ; p<.004)$ but the same speed as the $-\mathrm{s}$ plural $(\mathrm{t}(24)=1.201 ; p<.241)$. No difference between the -er and - s plural was observed $(\mathrm{t}(24)=-.827 ; p=.416)$. To summarize, the following reaction time pattern was observed (from fastest to slowest): -(e)n $<-\mathrm{e}<-$ er; with the -s plural not differing significantly from any of the other categories (see Figure 1).

Comparing the correct and incorrect plurals, the responses to the correct plurals are mostly processed faster than to the incorrect (-er versus -er violated by -e: $p<.001$; e versus -e violated by -er: $p<.001$ ). An exception was the -(e)n violated by -s condition, where the incorrect forms were processed faster that the correct items $(\mathrm{t}(24)=3.750 ; p<.001)$. The difference between the cross-condition $-\mathrm{s}$ and $-\mathrm{s}$ violated by $-(\mathrm{e}) \mathrm{n}$, however, was not significant $(\mathrm{t}(24)=.199 ; p=.844)$.

\subsection{Summary of the Behavioral Results}

Overall, the results show differences in the processing of the different plural categories. The reaction times for the different correct plural types differ, with -(e)n being the fastest and -er being the slowest (-(e)n $<-e=-s<$

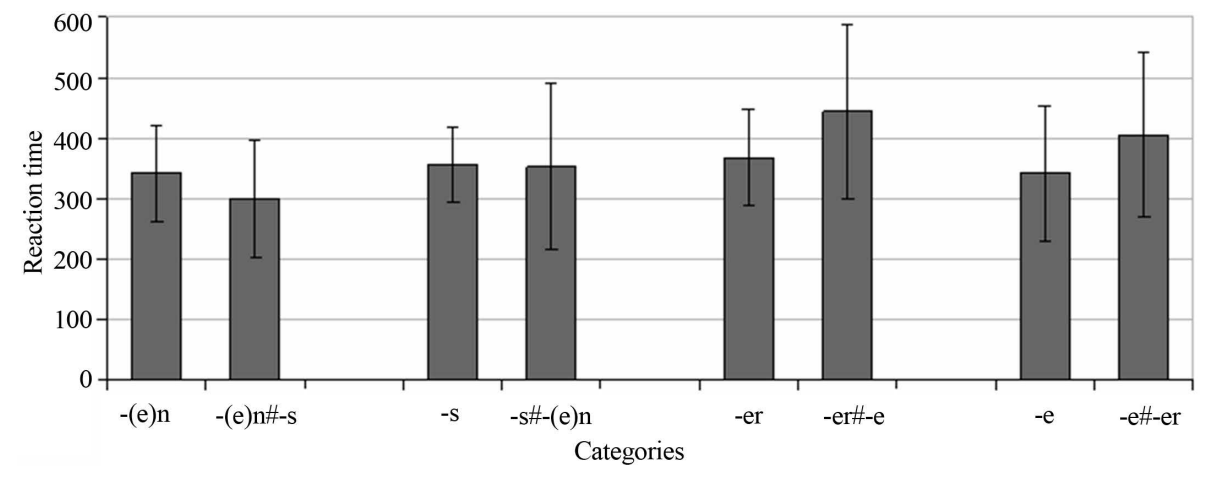

Figure 1. Mean reaction times to the correct (-(e)n, -er, -e, -s) and corresponding incorrect conditions (-(e)n\#-s, -er\#-e, -e\#-er,- s\#-(e)n) of experiment 1 with standard deviation bars. 
-er). The incorrect plurals are typically processed slower or at the same speed with the exception of -(e)n violated by -s being faster than the correct -(e)n plural.

All in all, the violation of the -(e)n category violated by -s differs from the other categories in the respect that the inverse pattern is found with regard to speed and accuracy of processing.

\section{Study II: ERP-Experiment}

\subsection{Procedure}

As the procedure was similar to the reaction time experiment, we will mainly focus on the differences between the studies. Subjects were instructed to press two buttons on a button box when they heard the item "Zwei Schirme" ("two umbrellas"), which functioned as a distractor to keep the subjects alert. The presentation and timing of the items was the same as in Study I. The total duration of the experiment was 35 minutes including breaks and 20 practice trials.

\subsection{EEG Recording and Data Analysis}

The EEG was recorded with active electrodes actiCAP and amplified with BrainAmp from 32 positions according to the 10 - 20-System (Jasper, 1958). Online filter was set to high cut-off of $250 \mathrm{~Hz}$ and a low cut-off of 0.1 $\mathrm{Hz}$.

The recorded data were averaged for each condition in every subject separately. The deviation point was set between the noun stem and the plural allomorph. The epochs started from $200 \mathrm{~ms}$ pre-deviation point to 2000 ms post-deviation point. Eye artifacts were corrected with ICA, noisy data were automatically removed and double-checked through visual inspection. Data were filtered offline with a low cutoff of $0.1 \mathrm{~Hz}$ and a high cutoff of $30 \mathrm{~Hz}$ before segmentation. Two participants were excluded due to excessive artifacts.

For statistical analysis, 27 electrodes were grouped into 9 regions of interest: left anterior (F7, F3, FC5), central anterior (FC1, Fz, FC2), right anterior (F4, F8, FC6), left medial (T7, C3, CP5), central medial (CP1, Cz, $\mathrm{CP} 2)$, right medial (C4, T8, CP6), left posterior (P7, P3, PO9), central posterior (O1, Pz, O2) and right posterior (P4, P8, PO10). The visual inspection of the ERP waveforms revealed rather slow changes of the ERP amplitude in all experimental conditions. To test for ERP amplitude differences across conditions, we calculated the mean amplitudes in four successive 200 ms time windows in the time range from 200 - 1000 ms post-deviation point.

\subsection{EEG Results}

Mean ERP-amplitudes were statistically analyzed using an ANOVA with the repeated measures factors plural type (-(e)n/-er/-e/-s), violation (correct/incorrect), hemisphere (left/center/right) and site (anterior/medial/posterior).

Only in an early time window (200 - $400 \mathrm{~ms})$, a significant interaction of plural category x violation $(\mathrm{F}(3,72)$ $=4.482 ; p=.011)$ was found but no main effect of violation $(\mathrm{F}(1,24)=.013 ; p=.910)$ (see Figure 2). Subsequently, violation effects were examined in the different plural categories in this early time window. A violation effect was observed for $-(\mathrm{e}) \mathrm{n}$ violated by $-\mathrm{s}(\mathrm{F}(1,27)=5.4, p=.029$ and for $-\mathrm{s}$ plural violated by $-(\mathrm{e}) \mathrm{n}(\mathrm{F}(1,27)=$ $8.0, p=.009$ ). For the two other conditions (-e plural violated by -er) and for the -er plural (violated by -e) no significant effects were found in this time window.

An analysis using dependent samples t-tests of the ROIs for the -n and -s conditions in the violated condition revealed a significant negative response for the left anterior region for both conditions (-n violated by $-\mathrm{s}: \mathrm{t}(24)=$ 2.756; $p<.011$ and $-\mathrm{s}$ violated by $-\mathrm{nt}(24)=3.111 ; p<.005$ ) as well as a right anterior negativity for $-\mathrm{n}$ violated by $-\mathrm{s}(\mathrm{t}(24)=-3.897 ; p<.001)$. A wide-spread positivity across several central and posterior ROIs was found for $-n$ violated by $-\mathrm{s}$ (e.g. centro-posterior parietal region (O1, Pz, O2): $-\mathrm{n}$ violated by $-\mathrm{s}$ : $\mathrm{t}(24)=-3.679409$; $p$ $<.00201$; -s violated by $-\mathrm{nt}(24)=2.323 ; p<.029)$ ). In the time window between $400-600 \mathrm{~ms}$, there was no effect of plural type but a main effect of violation $(\mathrm{F} 1,24)=5.122 ; p<.033)$. In the time window between 600 $800 \mathrm{~ms}$ and $800-1000 \mathrm{~ms}$, there was no interaction plural type/violation and no main effect of violation found.

\section{Discussion}

The current study demonstrated differences as well as similarities between the plural categories in both the RTas well as in ERP-experiments, even though the patterns did not necessarily overlap. The pattern that was found 

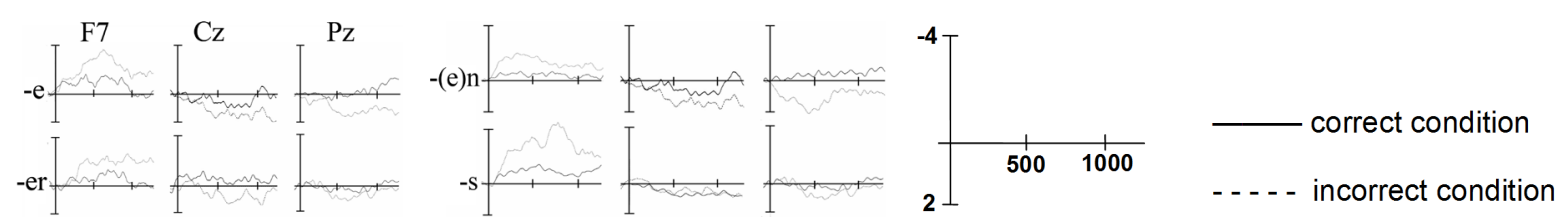

Figure 2. Event-related brain potentials (ERP)s to the categories (-(e)n, -er, -e, -s) in the electrodes F7 (left column), Cz (middle column) and $\mathrm{Pz}$ (right column). Correct condition is represented by the black line, incorrect conditions by dotted line. Horizontal scale: Tickmark at 500 and $1000 \mathrm{~ms}$, vertical line at $0 \mathrm{~ms}$ (deviation point onset): -4 to $+2 \mu \mathrm{V}$.

for the RT-experiment with -(e)n violated by -s behaving in an inverse manner compared to the other plural categories was not confirmed in the ERP-experiment. Here, the -(e)n and its violation with -s showed the strongest violation effect at frontal and centro-parietal electrodes. Also, while in the RT-experiment differences between -s and its violation with -(e)n could not be found, the ERP-experiment showed a clear violation effect. In the ERP-experiment, -e violated by -er and the cross-condition did not yield any results in the early time window while in the RT-experiment there were statistically significant differences between the correct and incorrect versions.

The special status of -s could only be partially confirmed. In the current RT-experiment the violation is processed faster than the correct forms. However, both -(e)n and -s behave more unexpectedly in the RT-experiment then -e and -er. In the ERP-experiment, as in the study by Lück et al. (2006) (and contrary to Weyerts et al. (1997)) the violation with -s and the violation with -(e)n evoked a significant and early response in frontal regions. However, in the current study, irregularization effects, as reflected by a central negativity (as in Bartke et al., 2005; Lück et al. (2006) (albeit a very small negativity)) and Weyerts et al. (1997), and with bilinguals in Hahne et al. (2006) could not be observed. Lück et al. (2006) and the current study presented the stimuli auditorily which may have contributed to these early effects found in the study by Lück and the current study across plural categories. As Lück et al. (2006) argue that unlike in visual presentation, the auditory modality words are usually not accessed as a whole but in a more serial fashion. As in the current study differences between -n and -s were most likely driven by co-articulation effects, this may also explain the results of Lück et al. (2006).

The ERP-results and the results of the RT-experiment suggest possibly different mechanisms at play here: It is viable the violation of an irregular plural form may elicit a different response than regular and irregular plural violated cross-wisely. How and whether these results are in line with the DMM-predictions cannot be determined at this point. It is also possible — and more likely_that phonetic salience plays a role here: Particularly the paradigm -e violated by -er and the cross-condition appear to function differently with or without focused attention. Moreover, -er and -e plurals allow Umlaut (such as ä, ö) whereas -n and -s plurals do not, which may have created more acoustic variance in those conditions.

In German, nouns with the -er plural and the -e are typically pronounced with a schwa-ending and may therefore be very difficult to discriminate from the -e suffix. Compared to this, -(e)n/-s plurals are acoustically more salient. Particularly in the paradigm -(e)n violated by -s, recognition of the violation appears to be greatly aided by co-articulation effects from the -n. It is possible that the recognition of the incorrect * "Affes" occurs earlier then recognition of the correct "Affen" if the - $n$ is missing.

All in all, future studies must clarify the role of acoustic salience effects in the processing of plural morphemes and their violations by closely controlling the stimuli used in the experiments. It also appears to be of high importance to include a wide range of plural categories in an experiment to obtain a more complete picture of plural processing.

\section{Acknowledgements}

This study was funded by the German Ministry of Education and Research (BMBF grant awarded to TR) and supported by the Zukunftskolleg at the University of Konstanz, as well as the German Research Foundation (DFG grant EU 39/7-1 awarded to CE).

We would like to thank Oleksiy Bobrov for the technical assistance and Elif Bamyac1, Ersin Abinik, Beyza Acar, Ramona Grutschnig, Pembe Güccuk, and Senia Mader for help with the subject recruitment and testing.

\section{References}

Bartke, S., Rösler, F., Streb, J., \& Wiese, R. (2005). An ERP-Study of “Irregular” Morphology. Journal of Neurolinguistics, 
18, 29-55. http://dx.doi.org/10.1016/j.jneuroling.2004.10.001

Clahsen, H. (1999). Lexical Entries and Rules of Language: A Multidisciplinary Study of German Inflection. Behavioral and Brain Sciences, 22, 991-1060. http://dx.doi.org/10.1017/S0140525X99002228

Hahne, A., Mueller, J. L., \& Clahsen, H. (2006). Morphological Processing in a Second Language: Behavioural and EventRelated Brain Potential Evidence for Storage and Decomposition. Journal of Cognitive Neuroscience, 18, 121-134. http://dx.doi.org/10.1162/089892906775250067

Jasper, H. H. (1958). The Ten-Twenty Electrode System of the International Federation. Electroencephalography and Clinical Neurophysiology, 10, 371-375.

Kluender, R., \& Kutas, M. (1993). Bridging the Gap: Evidence from ERPs on the Processing of Unbounded Dependencies. Journal of Cognitive Neuroscience, 5, 196-214. http://dx.doi.org/10.1162/jocn.1993.5.2.196

Kutas, M., \& Hillyard, S. A. (1980). Reading Senseless Sentences: Brain Potentials Reflect Semantic Incongruity. Science, 207, 203-205. http://dx.doi.org/10.1126/science.7350657

Luce, R. D. (1986). Response Times. New York: University Press.

Lück, M., Hahne, A., \& Clahsen, H. (2006). Brain Potentials to Morphologically Complex Words during Listening. Brain Research, 1077, 144-152. http://dx.doi.org/10.1016/j.brainres.2006.01.030

Marcus, G. F., Brinkmann, U., Clahsen, H., Wiese, R., \& Pinker, S. (1995). German Inflection: The Exception That Proves the Rule. Cognitive Psychology, 29, 189-256. http://dx.doi.org/10.1006/cogp.1995.1015

Oldfield, R. C. (1971). The Assessment and Analysis of Handedness: The Edinburgh Inventory. Neuropsychologia, 9, 97113. http://dx.doi.org/10.1016/0028-3932(71)90067-4

Penke, M., \& Krause, M. (2002). German Noun Plurals_A Challenge to the Dual-Mechanism Model. Brain and Language, 81, 303-311. http://dx.doi.org/10.1006/brln.2001.2526

Pinker, S., \& Prince, A. (1992). Regular and Irregular Morphology and the Psychological Status of Rules of Grammar. Proceedings of the 17th Berkeley Linguistics Society, USA, 230-251.

Ratcliff, R. (1993). Methods for Dealing with Reaction Time Outliers. Psychological Bulletin, 114, 510-532. http://dx.doi.org/10.1037/0033-2909.114.3.510

Rinker, T., Budde, N., Bamyaci, E., \& Winter, V. (2011). Einblicke in die Sprachlichen Leistungen Türkischer Kinder mit Deutsch als Zweitsprache. Diskurs Kindheits- und Jugendforschung, 6, 471-478.

Weyerts, H., Penke, M., Dohrn, U., Clahsen, H., \& Münte, T. (1997). Brain Potentials Indicate Differences between Regular and Irregular German Plurals. Neuroreport, 8, 957-962. http://dx.doi.org/10.1097/00001756-199703030-00028

Wiese, R. (2009). The Grammar and Typology of Plural Noun Inflection in Varieties of German. Journal of Comparative Germanic Linguistics, 12, 137-173. http://dx.doi.org/10.1007/s10828-009-9030-z 